\title{
Crystal structure of a PPR1-DNA complex: DNA recognition by proteins containing a $\mathrm{Zn}_{2} \mathrm{Cys}_{6}$ binuclear cluster
}

\author{
Ronen Marmorstein ${ }^{1}$ and Stephen C. Harrison ${ }^{2}$ \\ Harvard University, Department of Biochemistry and Molecular Biology, and ${ }^{2}$ Howard Hughes Medical Institute, \\ Cambridge, Massachusetts 02138 USA
}

PPR1 is a yeast transcription factor that contains a six-cysteine, two-zinc (Zn) domain, homologous to a similar structure in GAL4. Like GAL4, it binds to DNA sites with conserved CGG triplets symmetrically placed near each end. Whereas the GAL4 site has 11 intervening base pairs, the PPR1 site has 6 . The crystal structure of a 95-residue fragment of PPR1 in specific complex with DNA shows that the protein binds to a symmetrical 14-bp recognition site as a nonsymmetrical homodimer. An amino-terminal Zn domain interacts with a conserved CGG triplet near each end of the site through major groove contacts, and the carboxy-terminal residues mediate dimerization through a coiled-coil element and an extended strand. A linker region, connecting the $\mathrm{Zn}$ domain and the coiled-coil, folds into a $\beta$-hairpin. This hairpin packs differently on the two subunits and leads to a striking asymmetry, which is largely restricted to the dimerization and linker regions of the protein. Comparison with the GAL4-DNA structure shows that their specificities for sites of different length are determined by the preferred folds of their respective linker segments and by residues at the amino-terminal ends of their coiled-coils. None of these residues contact DNA in PPR1, and they contact only the sugar phosphate backbone in GAL4. We propose that this novel mode of DNA site selection is employed by other proteins that contain a $\mathrm{Zn}_{2} \mathrm{Cys}_{6}$ binuclear cluster.

[Key Words: PPR1; GAL4; zinc finger; $\mathrm{Zn}_{2} \mathrm{Cys}_{6}$ binuclear cluster; transcription factor; DNA recognition]

Received June 28, 1994; revised version accepted August 30, 1994.

The yeast protein PPR1 activates transcription of the genes URA1, URA3, and URA4, which encode enzymes involved in the regulation of pyrimidine levels (Loison 1980; Guyonvach 1986). Regulation is mediated by the binding of PPR1 to a 14-bp sequence located upstream of these genes (Roy et al. 1990). PPR1 is one of at least 12 fungal transcription factors that contain a six-cysteine, two-zinc $(\mathrm{Zn})$ domain, sometimes called a $\mathrm{Zn}_{2} \mathrm{Cys}_{6}$ binuclear cluster (Fig. 1a; for review, see Johnston 1987; Andre 1990). DNA targets for several members of this family have two symmetrically placed CGG elements, separated by a spacing characteristic of the particular protein that recognizes the site (Bram and Kornberg 1985; Giniger et al. 1985; Breunig and Kuger 1987; Leonardo et al. 1987; Ruzzi et al. 1987; Friden and Schimmel 1988; Siddiqui and Brandriss 1989; Roy et al. 1990; Fig. $1 \mathrm{~b})$. For example, GAL4 binds to $5^{\prime}-\mathrm{CGG}\left(\mathrm{n}_{11}\right) \mathrm{CCG}$, and PPR1 binds to $5^{\prime}-\mathrm{CGG}\left(\mathrm{n}_{6}\right) \mathrm{CCG}$ (Fig. 2). The structure of the DNA-binding domain of GAL4 in complex with a consensus DNA target (Marmorstein et al. 1992; Fig. $3 \mathrm{~b})$ shows that the protein attaches to its DNA site

\footnotetext{
${ }^{1}$ Present address: The Wistar Institute and the Department of Chemistry, University of Pennsylvania, Philadelphia, Pennsylvania 191044268 USA.
}

as a symmetrical dimer. The $\mathrm{Zn}_{2} \mathrm{Cys}_{6}$ domains at the amino-terminus of each subunit lie in the major groove and contact the two CGG elements. An extended 9-residue linker connects the $\mathrm{Zn}$ domain to a coiled-coil dimerization element, which rests over the minor groove in the center of the site. The presumed preference of the linker for its extended conformation and the observed electrostatic interactions between the DNA backbone and residues in the linker and amino-terminal tip of the coiled-coil appear to specify the 11-bp separation between the CGG triplets.

To understand better how two closely related proteins can distinguish between alternative spacing of conserved DNA elements, we have chosen to study the complex of PPR 1 (29-123) - a 95-residue, DNA-binding fragment of PPR1 and a specific DNA (Fig. 2). The sequence of the 14-bp synthetic DNA is a palindromic consensus of the four known PPRl sites (Keegan 1989; Roy et al. 1990). The crystal structure, determined to a nominal resolution of $3.2 \AA$, shows that the PPR $1 \mathrm{Zn}$ domains interact with CGG triplets just like those of GAL4 and that the linker segments, similar in number of amino acids to those of GAL4, fold up to fit the shorter site (Fig. 3b). The core of the dimerization element of PPR1 is a coiled-coil that fits asymmetrically against the linkers. The asym- 


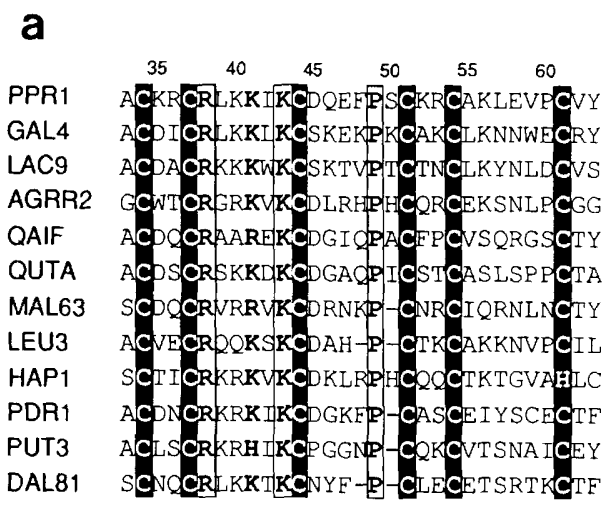

b

\begin{tabular}{|c|c|c|c|c|}
\hline & $\begin{array}{l}\mathrm{Zn}_{2} \mathrm{Cys}_{6} \\
\text { Region }\end{array}$ & Linker & $\begin{array}{c}\text { Heptads } \\
\text { (Dimerization) }\end{array}$ & DNA Site \\
\hline GAL4 & $10 \longrightarrow 40$ & (14) & $54-71$ & CGG $\left(N_{11}\right)$ CCG \\
\hline PPR1 & $33 \longrightarrow 63$ & (14) & $77-94$ & $\operatorname{CGG}\left(N_{6}\right) \operatorname{CCG}$ \\
\hline LEU3 & $36-69$ & (16) & $85-102$ & $\operatorname{CGG}\left(\mathrm{N}_{2}\right) \mathrm{CCG}$ \\
\hline JT3 & $33-62$ & (15) & $77 \longrightarrow 94$ & CGG $\left(N_{10}\right)$ CCG \\
\hline
\end{tabular}

Figure 1. Proteins that contain a $\mathrm{Zn}_{2} \mathrm{Cys}_{6}$ binuclear cluster. $(a)$ Homology among the fungal proteins. Conserved cysteine residues are shaded; other strictly conserved residues are boxed. Lys-41 of PPR 1, which is the only side chain that makes contacts to the functional groups of the DNA bases, is indicated in bold. (b) PPR 1 homologs that are known to bind DNA elements that contain inverted CGG half-sites. The $\mathrm{Zn}_{2} \mathrm{Cys}_{6}$ region starts 1 residue before the first conserved cysteine and 2 residues after the last conserved cysteine. The heptad region starts at the first heptad residue and ends at the last heptad residue. LAC9, the $K$. lactis homolog of GAL4, which binds the same DNA site, is omitted for clarity.

metric position of the coiled-coil, and the corresponding asymmetric fold of the linkers, creates on the surface of the complex a cavity that might serve as a binding site for some other component of the transcription machinery. Comparison of the PPR 1 and GAL4 complexes shows that corresponding protein elements-the $\mathrm{Zn}$ domain, the linker, and the coiled-coil-can be organized quite differently in the final three-dimensional structure of a DNA complex.

\section{Results}

Crystals of PPR1(29-123) complexed to a 14-bp consensus DNA site form in space group I222. The crystals showed a significant anisotropic falloff of the diffracted intensities at ambient temperatures, extending to a resolution of $3.2 \AA$ in the $\mathrm{b}^{\star}$ and $\mathrm{c}^{\star}$ directions but only 6.0 $\AA$ in the $\mathrm{a}^{*}$ direction. At $110^{\circ} \mathrm{K}$, accurate data can be obtained to $3.0 \AA$ in the $\mathrm{b}^{*}$ and $\mathrm{c}^{*}$ directions and $3.8 \AA$ in the $\mathrm{a}^{\star}$ direction. Thus, all data were collected at $110^{\circ} \mathrm{K}$, using a nitrogen stream to cool the crystals. The struc- ture was solved to $3.2 \AA$ by multiple isomorphous replacement (MIR), using four heavy atom derivatives, and refined to $3.2 \AA$ resolution as described in Materials and methods and Table 1. A representative region of the initial MIR map is shown in Figure 4.

\section{Structure of the PPR1/DNA complex}

The DNA in the PPR 1 complex conforms closely to the standard B-form. The average helical twist is $35.2^{\circ}$, and the mean rise per base pair is $3.36 \AA$. The 6 -bp central spacing places the two CGG elements almost precisely one turn apart in the major groove, and the protein therefore lies entirely against one face of the double helix (Fig. $3 \mathrm{a}, \mathrm{c})$. The two $\mathrm{Zn}$ domains, which contact the CGG triplets, lie above the major groove and nearly touch each other. The folded linkers are asymmetrically disposed and are distal to the DNA. The dimerization structure is perched on top of one of the two linkers, and the carboxy-terminal extensions drape over the coiled-coil toward the $\mathrm{Zn}$ domain of the apposing subunit.

The $\mathrm{Zn}$ domain of PPR1 (Fig. 5) is nearly identical in its structure to the homologous domain of GAL4 (Baleja et al. 1992; Kraulis et al. 1992; Marmorstein et al. 1992). When the $\alpha$ carbons of residues $10-40$ in GAL4 are superposed on those of residues 33-63 of PPR 1 , the root mean square (rms) deviation is only $0.8 \AA$ (Fig. 6b). There are two short helices (residues $34-41$ and 51-58), each followed by a sharp turn and an extended strand. An internal dyad relates one helix-strand segment (34-46) to

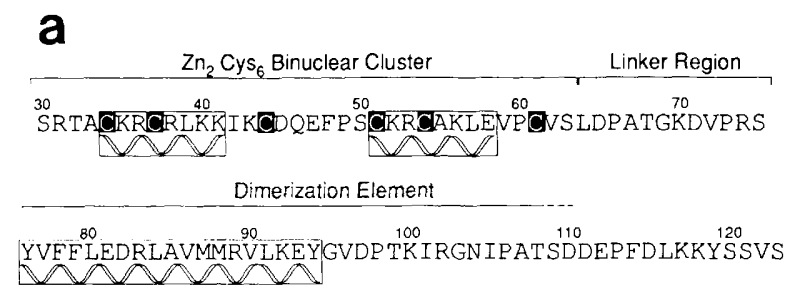

b

Consensus PPR 1

$\begin{array}{lllllllllllllllll}\text { Consensus } & \\ \text { Binding Site (4 sites): } & \text { T } & T & C & G & G & N & N & N & T & N & N & C & C & G & A & A \\ A & A & G & C & C & N & N & N & A & N & N & G & G & C & T & T\end{array}$

Oligonucleotide

In Cocrystal:

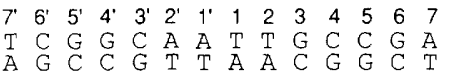

Figure 2. PPRl DNA-binding domain and its DNA-binding site. (a) Amino acid sequence of the DNA-binding fragment of PPR 1 used in the crystallographic analysis reported here. The various structural segments of the protein fragment are labeled above the sequence. Cysteine residues involved in chelating metal are shown in boldface type, and helical regions are indicated by wavy lines below the sequence. $(b)$ (Top) Consensus PPRl-binding site containing two highly conserved TCGG elements near the ends of the site. (Bottom) A 14-bp PPR 1-binding site used in the crystallographic analysis, with the numbering scheme used in the text indicated above the corresponding base. 

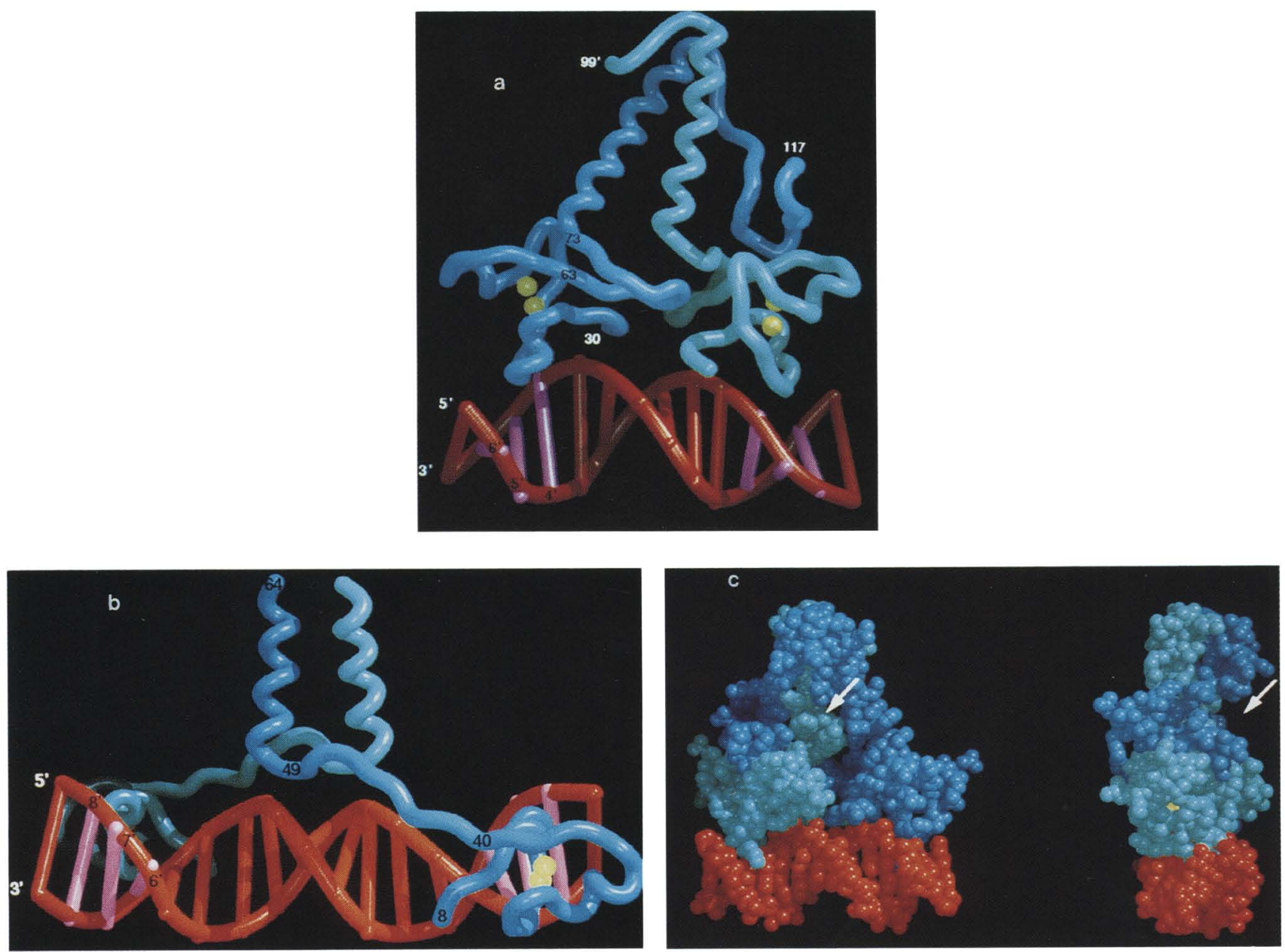

Figure 3. Structures of the PPR1-DNA and GAL4-DNA complexes. (a) PPR1-DNA complex viewed approximately normal to the twofold axis of the symmetrical parts of the complex. Amino acid sequence numbers at the borders of the unprimed protein segments ( $\mathrm{Zn}$ domain, residues 30-63; linker region, residues 64-72; dimerization region, residues 73-117) are shown. The carboxy-terminal end of the primed subunit is also indicated. The DNA is schematized as a helical ladder using C3' positions with the contacted CGG triplet DNA sequences labeled and highlighted in purple. The positions of the bound metal ions are shown as yellow balls. (b) GAL4-DNA complex viewed normal to the twofold axis of the complex. Amino acid sequence numbers at the borders of the three protein segments (Zn domain, residues 8-40; linker region, residues 41-49; and dimerization element, residues 50-64) are shown on one subunit. The contacted CGG triplet DNA sequences are labeled and highlighted in purple. (c) Space-filling representation of the PPR1-DNA complex highlighting the cavity on the surface of the protein (arrow). (Left) View is opposite to the direction in $B$; (right) view is down the DNA helical axis.

the other (51-63), even though there is no sequence identity except for the conserved cysteines. Moreover, the same dyad relates the two $\mathrm{Zn}$ ions. Thus, the three cysteines of each helix strand are all ligands for one $\mathrm{Zn}$, and the central cysteine is also a ligand for the other $\mathrm{Zn}$, completing a tetrahedral coordination shell. The overall twofold symmetry of the structure suggests that the domain may have evolved by duplication.

Residues in the carboxy-terminal half of this PPR 1 fragment provide the principal contacts stabilizing the dimer (Fig. 6a). A short $\alpha$-helical region forms a prototypical coiled-coil that is the central dimerization element. Coiled-coils facilitate dimerization in many other eukaryotic transcription factors (Landschulz et al. 1988; Murre et al. 1989; Jones 1990; O'Shea et al. 1991; Marmorstein et al. 1992). The coiled-coil in PPRl sits asym- metrically with respect to the DNA half-sites and the two $\mathrm{Zn}$ domains, and the two helices are of different length. One begins at Pro-73; the other at Ser-75'. Both end at Gly-95. Because of the asymmetry, we use primed numbers for one subunit and unprimed numbers for the other. The coiled-coil interface is typically hydrophobic, with a valine and two leucines at " $\mathrm{a}$ " positions and a leucine, methionine, and tyrosine at " $\mathrm{d}$ " positions. The outer surface of the coiled-coil is partly covered by the carboxy-terminal extended strands, which each lie against the helix of the opposite subunit. The two strands have somewhat different conformations; the ordered part of one extends to residue 117 , and the other only to residue $99^{\prime}$. Residues in the primed extension also refine with higher thermal parameters. Regions that are disordered in crystal structures are often flexible 
Table 1. Summary of crystallographic analysis

\begin{tabular}{|c|c|c|c|c|c|c|c|c|c|}
\hline & $\begin{array}{c}\text { Native } 1 \\
(W T)^{\mathrm{a}}\end{array}$ & $\begin{array}{c}\text { Native } 2 \\
\text { IU } 22^{\prime b}\end{array}$ & $\mathrm{IU} 121^{\prime} 2^{\prime \mathrm{b}}$ & IU $22^{\prime b}$ & $\mathrm{IC} 33^{\prime b}$ & $\mathrm{Hg}^{2+}$ & $\mathrm{TS}^{\mathrm{c}}$ & WS $^{\mathrm{d}}$ & $\mathrm{FS}^{e}$ \\
\hline \multicolumn{10}{|l|}{ Derivatives } \\
\hline resolution $(\AA)$ & 3.2 & 3.0 & 3.2 & 3.2 & 3.2 & 3.2 & & & \\
\hline redundancy & 5.4 & 4.6 & 2.5 & 2.0 & 2.9 & 3.9 & & & \\
\hline data coverage $(\%)$ & 93 & 98 & 91 & 75 & 63 & 98 & & & \\
\hline $\mathbf{R}_{\text {sym }}(\%)^{\mathrm{f}}$ & 9.3 & 6.4 & 6.3 & 5.8 & 4.6 & 7.0 & & & \\
\hline \multicolumn{10}{|l|}{ MIR analysis } \\
\hline resolution $(\AA)$ & & & $10-3.2$ & $10-3.2$ & $10-3.2$ & $10-3.2$ & & & \\
\hline mean isomorphous difference ${ }^{\mathrm{g}}$ & & & 19.2 & 16.1 & 21.4 & 21.0 & & & \\
\hline phasing power ${ }^{\mathrm{h}}$ & & & 1.13 & 0.89 & 0.83 & 1.73 & & & \\
\hline mean figure of merit & & & & 0.58 (overall) & & & & & \\
\hline \multicolumn{10}{|l|}{ Refinement } \\
\hline resolution $(\AA)$ & & & & & & & $10-3.2$ & & \\
\hline $\mathrm{R}$ factor $(\%)$ & & & & & & & & 24.5 & 33.0 \\
\hline reflections (all F) & & & & & & & 7096 & 6423 & 673 \\
\hline total number of atoms & & & & & & & 1826 & & \\
\hline rms bond length ${ }^{i}$ & & & & & & & 0.017 & & \\
\hline rms bond angle (deg. $)^{i}$ & & & & & & & 3.7 & & \\
\hline rms B values $\left(\AA^{2}\right)^{j}$ & & & & & & & 2.2 & & \\
\hline
\end{tabular}

a(WT) Wild type.

b(IU or IC) 5-Iodo-uridine or 5-iodo-cytosine has replaced thymine or cytosine, respectively, at the positions indicated (see Fig. $2 \mathrm{~b}$ ). '(TS) Total data set.

d(WS) Working data set containing $90 \%$ of randomly distributed data.

e(FS) Free data set containing $10 \%$ of randomly distributed data.

${ }^{f} R_{\text {sym }}=\Sigma_{h} \Sigma_{i}\left|I_{h, i}-I_{h}\right| / \Sigma_{h} \Sigma_{i} I_{h, i}$, where $I_{h}$ is the mean intensity of the $i$ observations of reflection $h$.

${ }^{g}$ Mean isomorphous difference $=\Sigma\left|F_{P H}-F_{P}\right| / \Sigma F_{P H}$, where $F_{P H}$ and $F_{P}$ are the derivative and native structure factor amplitudes, respectively.

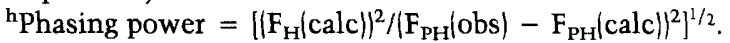

'The rms bond length and $\mathrm{rms}$ bond angle are the respective rms deviations from ideal values.

'The rms in B values is the rms deviations in temperature factors between bonded atoms.

in solution, and it is likely that the carboxy-terminal part of this fragment may serve as a flexible connection to other functional domains of the protein, such as transcriptional activation regions (Ptashne and Gann 1990).

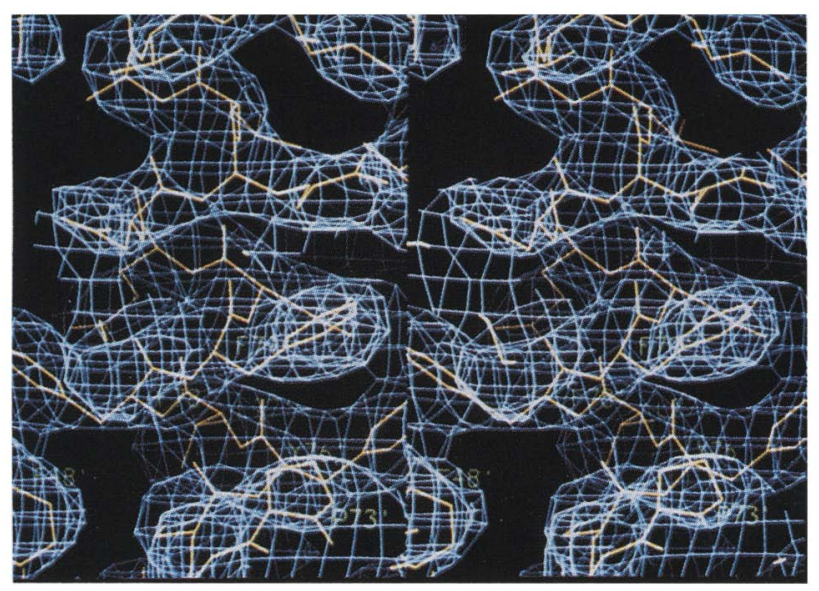

Figure 4. MIR electron density map of the complex. Stereoscopic view of the hydrophobic base of a helix comprising the coiled-coil dimerization element of the protein. The map was calculated to $3.2 \AA$ resolution after solvent flattening with SQUASH. The map is contoured at $1 \sigma$.
The segment we call the linker /residues 64-72 in one subunit, $64^{\prime}-74^{\prime}$ in the other) joins the $\mathrm{Zn}$ domain to the dimerization element. In GAL4, the linker is completely extended and makes essentially no contacts with other parts of the protein (Marmorstein et al. 1992). In PPR1, it folds into an antiparallel $\beta$-ribbon, with a 4-residue loop from Pro-66 to Gly-69 at its tip. Although the two linkers of the PPR1 dimer have essentially the same conformation (rms of main chain atoms of $0.74 \AA$ ), they are oriented differently with respect to the rest of the molecule. These alternative orientations are attributable largely to a $45^{\circ}$ difference between the psi torsion angles of residues 62 and $62^{\prime}$ (Fig. $6 \mathrm{~b}$ ). The linker of the unprimed subunit extends across the gap between the two $\mathrm{Zn}$ domains, whereas the linker of the primed subunit twists away from it. The unprimed linker thus forms a cradle against which the dimerization structure rests.

The amino-terminal end of the coiled-coil /Figs. 3 and 6a) is unusually hydrophobic. It sits above the unprimed linker. One helix lies over Leu-62 and Leu-72 at the base of the $\beta$ ribbon; the other helix lies over a hydrophobic patch (residues 66-68) at its tip. In addition, the coiledcoil is flanked by Phe- 48 and Pro- 49 from both zinc domains, as well as by Ile-101, and Ile-105 in the extended strands (Fig. 6a). 

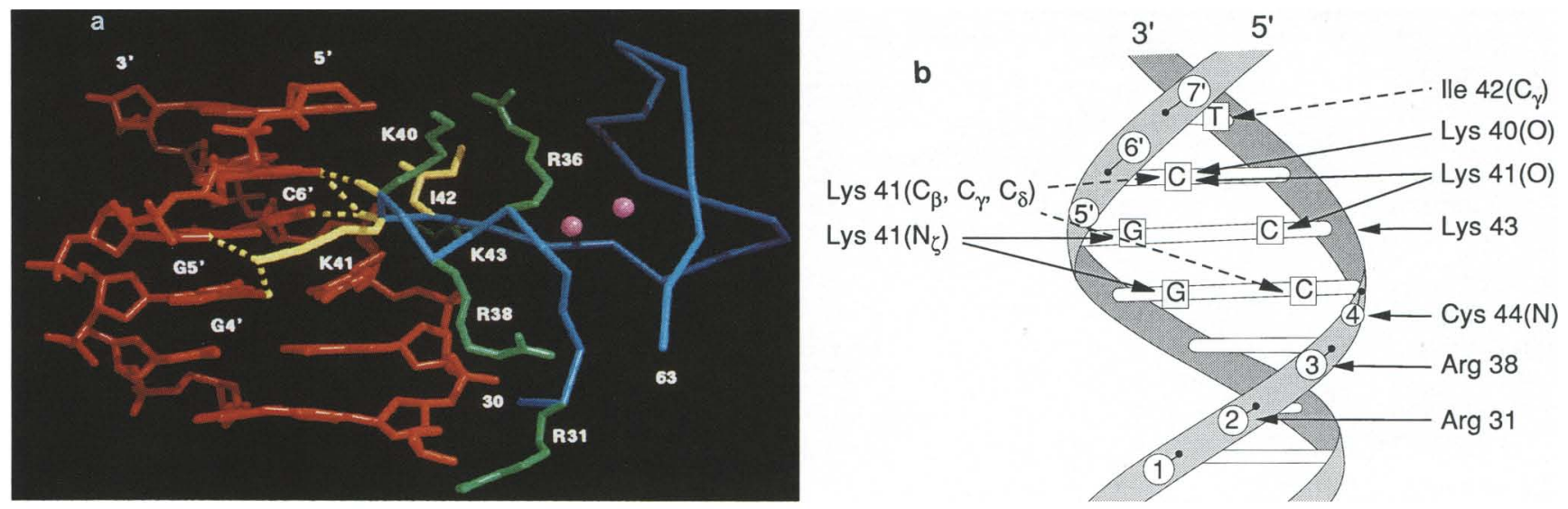

Figure 5. Structure of the $\mathrm{Zn}$ domain and its interaction with DNA. (a) Protein-DNA contacts mediated by the Zn domain. For clarity, only the $\mathrm{C}$ tracing of the protein (blue) is shown, except for the amino-acid side chains (green) that interact with DNA phosphate oxygens (red). Protein residues that make contacts to functional groups on the DNA bases are shown in yellow. Broken lines indicate hydrogen bonds between protein and DNA bases. Lys-40 could make a hydrogen bond with a phosphate on a longer DNA fragment. The metal ions are indicated by lavender balls. $(b)$ Schematic diagram showing all phosphate and base contacts with protein on the unprimed side of the complex. Identical contacts are made on the primed side except that the hydrogen bonds between the N7 of Lys- $41^{\prime}$ with the N7 of Gua 5', and O6 of Gua 4' are long $(3.7 \AA)$. Van der Waals contacts are indicated with broken lines.

\section{Interaction of PPR1 with DNA}

The consensus PPR1-binding site is a 14-bp sequence with two highly conserved TCGG elements in inverted orientation, separated by 6 intervening base pairs (Roy et al. 1990; see Fig. 2b for sequences and numbering convention). The PPR1 recognition modules are symmetri- cally anchored over the TCGG elements by salt bridges from a number of positively charged side chains and by a hydrogen bond from the peptide nitrogen of Cys-44 (Fig. 5). In addition to the contacts actually seen in the crystal, the side chains of Arg-36 and Lys-40 could form salt bridges with phosphates on more extended DNA segments. The carboxy-terminal end of the first helix in the
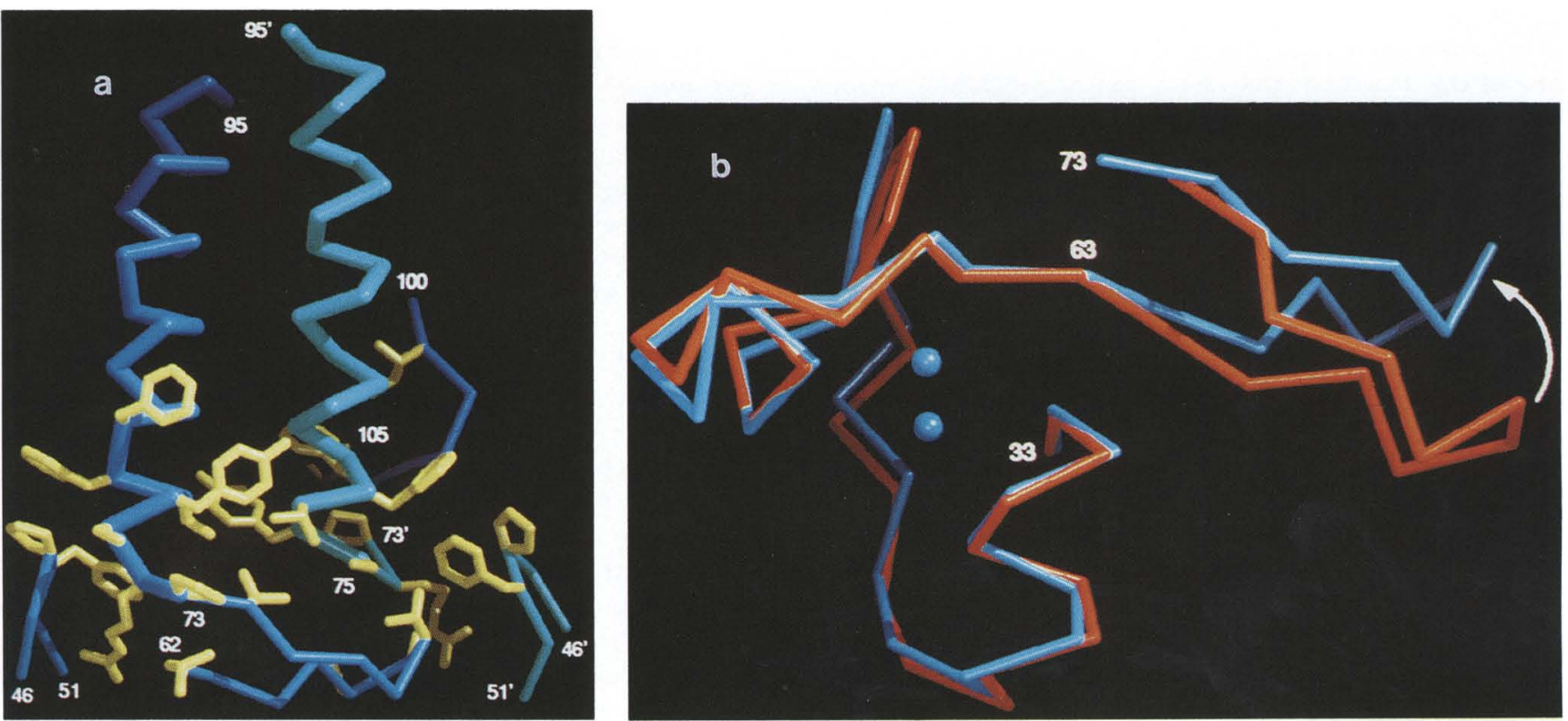

Figure 6. Asymmetry of the PPR1-DNA complex. $(a)$ Dimer interface of the PPR l-DNA complex. $\mathrm{C}_{\alpha}$ trace of residues near the dimer interface are shown in dark blue for the unprimed subunit and light blue for the primed subunit. Residues that contribute to the hydrophobic protein region stabilizing the dimer are shown in yellow. The heptad repeat of hydrophobic residues that stabilize the coiled-coil are not shown for clarity. (b) Comparison of the $\mathrm{Zn}_{2} \mathrm{Cys}_{6}$ and linker regions of the two subunits of the PPR1 homodimer using only the $\mathrm{Zn}_{2} \mathrm{Cys}_{6}$ regions (residues 33-63) for the superposition. The residues that begin and end the $\mathrm{Zn}_{2} \mathrm{Cys} \mathrm{s}_{6}$ and linker regions of the unprimed subunit (blue) are indicated. The $\mathrm{Zn}$ ions of the unprimed subunit are shown as blue balls. 
Zn domain points into the major groove. Lys-41, in the turn that follows this helix, makes a series of interactions with conserved bases. Its backbone carbonyl accepts hydrogen bonds from N4 of Cyt 5 and Cyt $6^{\prime}$; the aliphatic part of the lysyl side chain is in van der Waals contact with C5 of Cyt 4 and Cyt $6^{\prime}$; and the $\mathrm{N} \zeta$ donates hydrogen bonds to N7 of Gua 5' and O6 of Gua 4'. Residues 40 and 42 also contribute to the interface. The backbone carbonyl of Lys- 40 accepts a hydrogen bond from N4 of Cyt 6', and the C $\gamma$ of Ile-42 is in van der Waals contact with the 5-methyl group of Thy 7'.

\section{Discussion}

\section{Asymmetry of the PPR1 homodimer on DNA}

The unexpected asymmetry of the linkers, carboxy-terminal strands, and the coiled-coil is the most striking feature of the PPRl homodimer. This asymmetry appears to be driven largely by a complementary subunit association. This association creates a hydrophobic core, stabilized by residues at the base of the coiled-coil, in the loop regions of the $\mathrm{Zn}_{2} \mathrm{Cys}_{6}$ domains, and in one of the two asymmetrically disposed linker regions. The linkers of both subunits have very similar conformations (rms of main chain atoms of $0.74 \AA$ ), but they are effectively hinged with respect to the $\mathrm{Zn}$ domain, about an axis linking the $\mathrm{C}_{\alpha}$ atoms of residues 63 and 73 (Fig. $6 \mathrm{~b}$ ). In effect, there is a $45^{\circ}$ rotation about psi- 62 relative to psi-62'. The asymmetry allows the unprimed linker to contribute to the hydrophobic core by packing against the base of the coiled-coil (Fig. 6a).

There is a crystal contact surface between two dimers involving the carboxy-terminal end of the coiled-coil and the extended strands. This interface, which is largely stabilized by van der Waals interactions, has a solvent excluded surface of $1200 \AA^{2}$. The influence of this crystal packing interaction on the observed asymmetry cannot be assesed directly. We believe, however, that a symmetric orientation would be unlikely in solution, because it would disrupt the highly complementary packing of hydrophobic residues and would require a helical unraveling / the unprimed helix of the coiled-coil being 2 residues longer then the primed helix).

The asymmetric PPR1-DNA complex has a cavity on the surface of the protein near the center of the DNAbinding site (Fig. 3c). The cavity is surrounded by the two $\mathrm{Zn}$ domains and the coiled-coil. One side is exposed to solvent. The significance of this cavity is not known; however, we suggest that it could be a binding site for another protein component of the transcription machinery. Alternatively, it could be a site of contact from another region of PPR1.

\section{$D N A$ recognition by $P P R 1$}

The crystal structure of the PPR1-DNA complex shows that there are two components to DNA recognition by PPR1: (1) Recognition of CCG triplet sequences at the ends of the DNA site by residues within the $\mathrm{Zn}_{2} \mathrm{Cys}_{6}$ binuclear cluster; and (2) specificity for a 6-bp spacing separating these elements resulting from the complementary subunit interface of the PPR1 homodimer.

Measurements of the binding of PPR1(29-123) in vitro to a variety of synthetic DNA targets confirm the important determinants of DNA recognition illustrated by the crystal structure (S. Liang and M. Ptashne, unpubl.). Any symmetrical modification of the bases in the CGG triplets decreases affinity by at least 100 -fold, whereas the central 6 bp can be altered essentially ad libitum. The terminal $\mathrm{T}$ in the TCGG consensus can also be modified without loss in binding affinity, suggesting that the contact of its methyl group with Ile- 42 is not of major significance. Shortening or lengthening of the central spacing between the CGG triplets by 1 bp reduces affinity by five- to eightfold. Additional biochemical and crystallographic analysis will be required to establish how PPR 1 can accommodate a DNA site with an altered spacing between half sites. Preliminary experiments suggest that on such sites the PPR 1 dimer binds specifically to one half site and nonspecifically to the other, leaving the spacing between domains essentially unaltered ( $\mathrm{S}$. Liang and M. Ptashne, unpubl.).

\section{Comparison with the GAL4-DNA complex}

Comparison of the GAL4-DNA and PPR1-DNA complexes shows a conserved set of contacts with the CGG element, for which the key features are the folded structure of the polypeptide backbone, two carbonyl oxygens, and a single lysyl side chain just carboxy-terminal to the first helix (Marmorstein et al. 1992). This lysine is conserved in all but one of the homologous proteins containing a $\mathrm{Zn}_{2} \mathrm{Cys}_{6}$ region (for review, see Johnston 1987; Andre 1990). The one exception, PUT3, (Brandiss and Magasanik 1979; Brandriss 1987) has a histidine in the corresponding position. Modeling suggests that the histidine could make compensatory interactions.

The comparison of GAL4 and PPR1 also shows quite different sorts of dimer relationships, although both are based on homologous coiled-coil elements. In GAL4, the linker region is in an extended conformation and uses several basic residues to anchor it to the DNA backbone. The amino termini of the helices in the coiled-coil are also positively charged, tailoring it to contact phosphates on the minor groove surface of the DNA. Thus, the linker and dimerization element of GAL4 make a complementary electrostatic interface with the minor groove at the DNA site, spacing the $\mathrm{Zn}$ domains by $11 \mathrm{bp}$ (Marmorstein et al. 1992). In PPR1, the linkers fold into $\beta$-hairpins, one of which forms the base for the helices of the coiled-coil. The hydrophobic amino termini of these helices are also stabilized by residues within the $\mathrm{Zn}$ domains and one of the two asymmetrically disposed carboxy-terminal extended strands. The resulting subunit association creates a structure that is more compact than GAL4 and that is compatible with a relatively short DNA-binding site with two CGG triplets separated by 6 bp. 
DNA recognition by other proteins containing $\mathrm{a} \mathrm{Zn}_{2} \mathrm{Cys}_{6}$ binuclear cluster

Among the 12 fungal DNA-binding proteins containing $\mathrm{Zn}_{2} \mathrm{Cys}_{6}$ domains, several are known to recognize DNA sites with CGG triplets in inverted orientation and with a spacing characteristic of the protein in question. In addition to GAL4 and PPR1, these include PUT3 (Brandriss and Magasanik 1979; Brandriss 1987), LEU3 (Zhou et al. 1987; Friden et al. 1988), and the Kluyveromyces lactis homolog of GAL4, LAC9 (Wray et al. 1987; Halvorsen et al. 1991). LAC9, which is extremely similar to GAL4 within its linker and dimerization, has naturally occurring sites with spacing identical to those for GAL4 (Wray et al. 1987; Halvorsen et al. 1991). We believe that these proteins will be found to have a similar mode of bipartite DNA recognition: CGG triplets contacted by conserved $\mathrm{Zn}$ domains, with their spacing specified by nonconserved dimerization structures. The properties of chimeric proteins containing elements of GAL4, PPR1, and PUT3 (Reece and Ptashne 1993) are consistent with conclusions that we have drawn from the GAL4 and PPR 1 complexes. The $\mathrm{Zn}$ domains can be interchanged among the three proteins without affecting specificity, as expected from the conserved features of these regions. Chimeras with only the linkers interchanged are inactive, whereas hybrid proteins containing a 19-residue interchange comprising the linker and the amino-terminal part of the coiled-coil are active and exhibit the specificity of the protein from which the insert was derived. The characteristic interactions between the amino-terminal part of the coiled-coil and the linker in the PPR 1 complex and between the amino terminus of the coiledcoil and the DNA backbone in the GAL4 complex imply that these segments must be interchanged together to create a functional protein.

Families of transcription factors that have conserved, or partly conserved, half-site recognition modules, and a mode of dimerization that determines the half-site spacing are actually remarkably common. The $\lambda$ and 434 repressors have homologous recognition domains bearing a Gln-Gln motif that specifies a $5^{\prime}$ adenine in their operator sequences but somewhat different dimer interactions in the two repressors determining that the adenines have 13 or 12 intervening base pairs, respectively (Pabo et al. 1990; Harrison 1992). Within the hormone receptor family, the retinoid X receptor (RXR) participates in recognition of at least three sequences with conserved half-sites but with different spacings; the heterodimeric partner of RXR dictates site preference, presumably by establishing a distinctive dimer interaction (Naar et al. 1991; Umesono et al. 1991; Bugge et al. 1992; Marks et al. 1992).

Not all transcription factors with $\mathrm{Zn}_{2} \mathrm{Cys}_{6}$ domains bind to sites with inverted CGG elements. For example, although the ARGRII (Messenguy et al. 1991; Dubois and Messenguy 1991; Rijcke et al. 1992) and HAP1 (Clavilier et al. 1976; Guarente et al. 1984; Verdiere et al. 1986; Prezant et al. 1987; Schneider and Guarente 1991) proteins both use $\mathrm{Zn}_{2} \mathrm{Cys}_{6}$ domains to recognize DNA, neither of their DNA target sites contain inverted CGG half-sites. Thus, there is likely to be at least one other possible set of precise contacts between this kind of domain and DNA. Moreover, two of the three known HAPl sites bear little resemblance to each other, suggesting additional variability in contacts for the same protein. Structures of the relevant complexes will probably be required to sort out these non-GAL4-like specificities.

\section{Materials and methods}

Expression and purification of PPR1(29-123)

PPR1(29-123), which contains the full DNA-binding domain, was overexpressed in Escherichia coli from a bacteriophage T7 RNA polymerase promoter and purified as follows. Cells were grown at $37^{\circ} \mathrm{C}$ to an $\mathrm{OD}_{595}$ of 0.6 and induced with $0.5 \mathrm{~mm}$ isopropyl- $\beta$-D-thiogalactopyranioside (IPTG) and $100 \mathrm{~mm} \mathrm{Zn/ac-}$ etate) ${ }_{2}$ for $2.5 \mathrm{hr}$. The collected cells were lysed in a low salt buffer (LSB) containing $20 \mathrm{~mm}$ HEPES (pH 7.5), $0.15 \mathrm{~mm} \mathrm{NaCl}$, $10 \mathrm{~mm} \mathrm{Zn}(\text { acetate })_{2}, 1.4 \mathrm{~mm} \beta$-mercaptoethanol, and $0.1 \mathrm{mg} / \mathrm{ml}$ of PMSF. The supernatant was dialyzed against LSB containing $10 \%$ glycerol (LSBG) and chromatographed on an S-Sepharose fast flow column (Pharmacia). Fractionation was achieved using a $0.15-0.7 \mathrm{M} \mathrm{NaCl}$ gradient in LSBG. Peak fractions, which eluted near the end of the salt gradient, were pooled and precipitated with a solution containing $0.5 \mathrm{mg} / \mathrm{ml}$ of ammonium sulfate. The precipitant was redissolved in LSBG and chromatographed by gel filtration FPLC using a Superdex-75 column (Pharmacia). Peak fractions were concentrated to $\sim 60 \mathrm{mg} / \mathrm{ml}$ using a Centricon-10 microconcentrator (Amicon). Protein purity was judged to be $>95 \%$ by SDS-gel electrophoresis using Coomassie blue staining.

\section{Crystallization of the PPR1-DNA complex}

Oligonucleotides and related heavy-atom derivatives were synthesized on a Milligen DNA synthesizer and purified by reversephase HPLC using a Dynamax 300 column (Rainin). Crystals of PPR1(29-123) (Fig. 2a), bound to a consensus 14-bp oligonucleotide (Fig. 2b), were obtained by vapor diffusion using $6-\mu$ l hanging drops containing $0.6 \mathrm{~mm}$ protein dimer, $0.7 \mathrm{~mm}$ DNA duplex, 7\% PEG 3500 (polyethylene glycol, average molecular mass $3.5 \mathrm{kD}$ ), 1\% MPD (2-methyl-2,4-pentanediol), $87.5 \mathrm{mM}$ $\mathrm{NaCl}, 62.5 \mathrm{mM} \mathrm{CaCl}_{2}, 50 \mu \mathrm{M} \mathrm{Zn}$ (acetate) $)_{2}$ and $10 \mu \mathrm{M}$ sodium cacodylate $(\mathrm{pH} 6.8)$, equilibrated with a reservoir containing

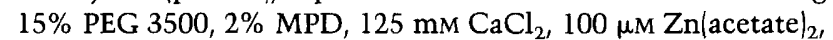
and $20 \mathrm{~mm}$ sodium cacodylate $(\mathrm{pH} 6.8)$. Crystals grew in several weeks to a typical size of $0.4 \times 0.4 \times 0.2 \mathrm{~mm}$. The crystals are in the space group $\mathrm{I} 222$, with $\mathrm{a}=53.8 \AA, \mathrm{b}=92.6 \AA$, and $\mathrm{c}=174.7 \AA$, when flash-frozen to $110^{\circ} \mathrm{K}$. All data were collected at $110^{\circ} \mathrm{K}$ using a Siemans area detector and $\mathrm{CuK}_{\mathrm{a}}$ radiation from an Elliott GX-13 generator with Frank mirrors. The data were processed with the program XDS (Kabsch 1988a,b).

\section{Structure determination}

The structure was determined at $3.2 \AA$ resolution using phase information from four derivatives-one $\mathrm{Hg}^{2+}$, two 5-iodo-uridine, and one 5-iodocytosine (see Fig. $2 b$ and Table 1). The heavy-atom coordinates demonstrated that there was one protein-DNA complex per asymmetric unit with a protein homodimer bound to one DNA duplex. The initial MIR map showed clear electron density for B-form DNA, for the $\mathrm{Zn}$ do- 
main of the protein (residues 33-63), and for a segment of a double-stranded coiled-coil. The program FRODO (Jones 1978) was used to place a model for the DNA based on idealized B-form coordinates and to position models derived from the homologous $\mathrm{Zn}$ domain of GAL4 on both half-sites in the complex. Residues 76-94 were modeled on coordinates from the coiled-coil of GCN4 (O'Shea et al. 1991). The sequence phase of the paired $\alpha$-helices was established by the presence of two adjacent tyrosine residues near their amino termini. The MIR map (Fig. 4) showed continuous density linking the $\mathrm{Zn}$ domain to the coiled-coil region, although some of the side chains were not clear. Also evident were $\sim 20$ carboxy-terminal residues from one subunit and 5 carboxy-terminal residues from the other. The electron density map was improved by combining model and MIR phases with the COMBINE program (Bricogne 1976) and solvent flattening with the program SQUASH (Zhang 1993). The model was adjusted further with reference to this modified map. The initial model excluded 10 carboxy-terminal residues on one subunit and 24 on the other. Two amino-terminal residues from each subunit were also not visible.

The initial model comprising residues $31-112$ of one protein subunit, 31-99 of the other, the four $\mathrm{Zn}$ ions, and the entire DNA molecule $(\sim 85 \%$ of the structure in all $)$ was refined against native diffraction amplitudes to $3.2 \AA$ resolution using CORELS (Sussman 1977) and XPLOR (Brünger et al. 1987; Brünger 1993). At this point an improved data set, which extended to $3.0 \AA$ in two directions and $3.8 \AA$ in the third, was obtained from an iodine derivative of the complex (Native 2), and this was used for all subsequent refinements (Table 1). A randomly selected $10 \%$ of the data were omitted from the refinement and used as a free data set to monitor subsequent calculations (Brünger 1992). To eliminate bias from the model, which had been refined against the previous native data set, the ideal structural units that had been used for the initial model were rigid-body fitted onto the partially refined coordinates, and the new model thus prepared was used in refinement against the working data set of Native 2. Conventional positional refinement to $3.2 \AA$ was then carried out with XPLOR (Brünger et al. 1987; Brünger 1992) initially using the following crystallographic constraints: (1) The coiled-coil, the metal-binding domain, and the DNA were individually constrained to local symmetry; (2) helical regions of the dimerization and $\mathrm{Zn}$ domains were tightly constrained to $\alpha$-helical backbone torsion angles; and (3) DNA backbone torsion angles were fixed to values determined previously in refinement cycles of CORELS (Sussman 1977). After an anisotropic B-factor correction, the constraints on structural symmetry were released and several more cycles of conventional refinement were carried out at $3.2 \AA$ resolution, followed by a tightly constrained individual atomic B-factor refinement. The correctness of the model was checked against simulated annealing omit maps (Hodel et al. 1992) over the entire structure, and the model was adjusted appropriately. A last round of refinement with the program TNT (Tronnud 1987) resulted in a model with good geometry and an $\mathrm{R}$ factor of $24.5 \%$ (free $\mathrm{R}$ factor $=33.0 \%$ ) using all reflections between 10 and $3.2 \AA$ (Table 1). A Ramachandran plot shows only two loop residues in disallowed regions.

\section{Acknowledgments}

We thank S. Liang and D. Liss for their contributions at the initial stages of the project; S. Liang, R. Reece and M. Ptashne for useful discussions and permission to site unpublished results; and N. Sinitskaya for synthesis and purification of oligunucleotides. This work was supported by a grant from the Na- tional Institutes of Health (NIH) (to M. Ptashne and S.C.H). R.M. acknowledges the NIH for a postdoctoral fellowship.

The publication costs of this article were defrayed in part by payment of page charges. This article must therefore be hereby marked "advertisement" in accordance with 18 USC section 1734 solely to indicate this fact.

\section{Note added in proof}

Coordinates of the PPR1/DNA complex will be deposited in the Protein Data Bank. They may also be obtained from the authors by request.

\section{References}

Andre, B. 1990. The UGA3 gene regulating the GABA catabolic pathway in Saccharomyces cerevisiae codes for a putative zinc finger protein acting on RNA levels. Mol. \& Gen. Genet. 220: 269-276.

Baleja, J.D., R. Marmorstein, S.C. Harrison, and G.Wagner. 1992. Solution structure of the DNA-binding domain of $\mathrm{Cd}_{2}$ GAL4 from Saccharomyces cerevisiae. Nature 356: 450-452.

Bram, R. and R.D. Kornberg. 1985. Specific upstream binding to far upstream activating sequences in polymerase II promoters. Proc. Natl. Acad. Sci. 82: 43-47.

Brandriss, M.C. 1987. Evidence for positive regulation of the proline utilization pathway in Saccharomyces cerevisiae. Genetics 117: 429-435.

Brandriss, M.C. and B. Magasanik. 1979. Genetics and physiology of proline utilization in Saccharomyces cerevisiae: Mutation causing constitutive enzyme expression. J. Bacteriol. 140: 504-507.

Breunig, K.D. and P. Kuger. 1987. Functional homology between the yeast regulatory proteins GAL4 and LAC9: LAC9mediated transcriptional activation in Kluyveromyces lactis involves protein binding to a regulatory sequence homologous to the GAL4 protein-binding site. Mol. Cell. Biol. 7: 4400-4406.

Bricogne, G. 1976. Methods and programs for direct space exploitation of geometric redundancies. Acta Crystallogr. A 32: 832-847.

Brünger, A.T. 1992. The free R value: A novel statistical quantity for assessing the accuracy of crystal structures. Nature 335: 472-474.

- 1992. XPLOR manual, version 3.1.A. 1993. Yale University, New Haven, CT.

Brünger, A.T., J. Kuriyan, and M. Karplus. 1987. Crystallographic $\mathrm{R}$ factor refinement by molecular dynamics. Science 235: 458-460.

Bugge, T.H., J. Pohl, O. Lonnoy, and G. Stunnenberg. 1992. RXR alpha, a promiscuous partner of retinoic acid and thyroid hormone receptors. EMBO /. 11: 1409-1418.

Clavilier, L., G. Pere Aubert, M. Somlo, and P.P. Slonimski. 1976. Network of interactions between unlinked genes: Synergistic and antagonistic regulation of iso-1-cytochrome $\mathrm{c}$, iso-2-cytochrome $\mathrm{c}$ and cytochrome $\mathrm{b} 2$ synthesis. Biochimie 58: $155-172$.

Dubois, E. and F. Messenguy. 1991. In vitro studies of the binding of the ARGR proteins to the ARG5,6 promoter. Mol. Cell. Biol. 11: 2162-2168.

Friden, P. and P. Schimmel. 1988. LEU3 of Saccharomyces cerevisiae activates multiple genes for branched chain amino acid biosynthesis by binding to a common decanucleotide core sequence. Mol. Cell. Biol. 8: 2690-2697.

Giniger, E., S.M. Varnum, and M. Ptashne. 1985. Specific DNA binding of GAL4, a positive regulatory protein of yeast. Cell 
40: $767-774$.

Guarente, L., B. Lalonde, P. Clifford, and E. Alani. 1984. Distinctly regulated upstream activation sites mediate catabolite repression of the CYC1 gene of Saccharomyces cerevisiae. Cell 36: 503-511.

Halvorsen, Y-D.C., K. Nandabalan, and P.C. Dickson. 1991. LAC9 DNA binding domain coordinates two zinc atoms per monomer and contacts DNA as a dimer. Mol. Cell. Biol. 11: $1777-1784$.

Harrison, S.C. 1992. Transcriptional regulation, (ed. K. Yamamoto and S. McKnight), pp. 449-473. Cold Spring Harbor Laboratory Press, Cold Spring Harbor, New York.

Hodel, A., S.-H. Kim, and A.T. Brunger. 1992. Model bias in macromolecular crystal structures. Acta Crystallogr. A48: $851-858$.

Johnston, M. 1987. A model fungal gene regulatory mechanism: The GAL4 genes of Saccharomyces cerevisiae. Microbiol. Rev. 51: 458-476.

Jones, N. 1990. Transcriptional regulation by dimerization: Two sides to an incestuous relationship. Cell 61:9-11.

Jones, T.A. 1978. A graphics model building and refinement system for macromolecules. J. Appl. Crystallogr. 11: 268272.

Kabsch, W. 1988a. Evaluation of single-crystal X-ray diffraction data from a position-sensitive detector. J. Appl. Crystallogr. 21: 916-924.

- $1988 \mathrm{~b}$. Automatic indexing of rotation diffraction patterns. J. Appl. Crystallogr. 21: 67-71.

Keegan, L. 1989. "Studies on the DNA-binding of the yeast GAL4 protein." $\mathrm{PhD}$. thesis. Harvard University, Cambridge, MA.

Kraulis, P.J., A.R.C. Raine, P.L. Gadhavi, and E.D. Laue. 1992. Structure of the DNA binding domain of zinc GAL4. Nature 356: $448-450$.

Landschulz, W. H., P.F. Johnson, and S.L. McKnight. 1988. The leucine zipper: A hypothetical structure common to a new class of DNA binding proteins. Science 245: 1759-1764.

Leonardo, J.M., S.M. Bhairi, and R.C.Dickson. 1987. Identification of upstream activator sequences that regulate induction of the beta-galactosidase gene in Kluyveromyces lactis. Mol. Cell. Biol. 7: 4369-4376.

Loison, G., R. Losson, and F. Lacroute. 1980. Constitutive mutants for orotidine 5 phosphate decarboxylase and dihydroorotic acid dehydrogenase in Saccharomyces cerevisiae. Curr. Genet. 2: 39-44.

Marks, M.S., P.L. Hallenbeck, T. Nagata, J.H. Segars, A. Ettore, V.M. Nikodem, and K. Ozato. 1992. H-2RIIBP (RXR beta) heterodimerization provides a mechanism for combinatorial diversity in the regulation of retinoic acid and thyroid hormone responsive genes. EMBO J. 11: 1419-1435.

Marmorstein, R., M. Carey, M. Ptashne, and S.C. Harrison. 1992. DNA recognition by GAL4: Structure of a proteinDNA complex. Nature 356: 408-414.

Messenguy, R.F., E. Dubois, and C. Boonchird. 1991. Determination of the DNA binding sequences of the ARGR proteins to arginine anabolic and catabolic promoters. Mol. Cell. Biol. 11: 2852-2863.

Murre, C., P.S. McCaw, and D. Baltimore. 1989. A new DNA binding and dimerization motif in immunoglobulin enhancer binding, daughterless, myoD, and myc proteins. Cell 56: $777-783$.

Naar, A.M., J-M. Boutin, S.M. Lipkin, V.C. Yu, J.M. Holloway, C.K. Glass, and M. Rosenfeld. 1991. The orientation and spacing of core DNA binding motifs dictate selective transcriptional responses to three nuclear receptors. Cell 65: $1267-1279$.
O'Shea, E.K., J.D. Klemm, P.S. Kim, and T. Alber. 1991. X-ray structure of the GCN4 leucine zipper, a two stranded parallel coiled-coil. Science 254: 539-244.

Pabo, C.O., A.K. Aggarwal, S.R. Jordan, L.J. Beamen, U.R. Obeysekare, and S.C. Harrison. 1990. Conserved residues make similar contacts in two repressor-operator complexes. Science 257: 1210-1213.

Prezant, T., K. Pfeifer, and L. Guarente. 1987. Organization of the regulatory region of the yeast CYC7 gene: Multiple factors are involved in regulation. Mol. Cell. Biol. 7:32523259.

Ptashne, M. and A.A.F. Gann. 1990. Activators and targets. Nature 346: 329-331.

Reece, R.J. and M. Ptashne. 1993. Determinants of binding site specificity among yeast $\mathrm{C}_{6}$ zinc cluster proteins. Science 261: 909-911.

Rijcke, M.D., S. Seneca, B. Punyammalee, N. Glansdorff, and M. Crabeel. 1992. Characterization of the DNA target site for the yeast ARGR regulatory complex, a sequence able to mediate repression of induction by arginine. Mol. Cell. Biol. 12: 68-81.

Roy, A., F. Exinger, and R. Lossen. 1990. Cis- and trans-acting regulatory elements of the yeast URA3 promoter. Mol. Cell. Biol. 10: 5257-5270.

Ruzzi, M., K.D. Breunig, A.G. Ficca, and C.P. Hollenberg. 1987. Positive regulation of the beta galactosidase gene from Kluyveromyces lactis is mediated by an upstream activation site of Saccharomyces cerevisiae. Mol. Cell. Biol. 7:991997.

Schneider, J.C. and L. Guarente. 1991. Regulation of the yeast CYT1 gene encoding cytochrome $\mathrm{cl}$ by HAP1 and HAP2/3/ 4. Mol. Cell. Biol. 11: 4934-4942.

Siddiqui, A.H. and M.C. Brandriss. 1989. The Saccharomyces cerevisiae PUT3 activator protein associates with proline specific upstream activator sequences. Mol. Cell. Biol. 9: $4706-4712$.

Sussman, J.L., S.R. Holbrook, G.M. Church, and S.H. King. 1977. A structure-factor least squares refinement procedure for macromolecular structures using constrained and restrained parameters. Acta Crystallogr. A33: 800-804.

Tronrud, D.E., L.F. Ten Eyck, and B.W. Matthews. 1987. An efficient general purpose least squares refinement program for macromolecular structures. Acta Crystallogr. A 43: 489501.

Umesono, K., K. Murakami, C.C. Thompson, and R.M. Evans. 1991. Direct repeats as selective response elements for the thyroid hormone, retinoic acid, and vitamin D3 receptors. Cell 65: 1255-1266.

Verdiere, J., F. Greusot, L. Guarente, and P. Slonimski. 1986. The overproducing CYP1 and the underproducing HAP1 mutations are alleles of the same gene which regulates in trans the expression of the structural genes encoding isocytochromes c. Curr. Genet. 10: 339-342.

Wray, L.U. Jr., M.M. Witte, R.C. Dickson, and M.I. Riley. 1987. Characterization of a positive regulatory gene, LAC9, that controls induction of the lactose-galactose regulone of Kluyveromyces lactis: Structural and functional relationships to GAL4 of Saccharomyces cerevisiae. Mol. Cell. Biol. 7: 1111-1121.

Zhang, J.Y.K. 1993. SQUASH-combining constraints for macromolecular phase refinement and extension. Acta Crystallogr. D49: 213-222.

Zhou, K., P.R.G. Brisco, A.E. Hinkkanen, and G.B. Kohlhaw. 1987. Structure of yeast regulatory gene LEU3 and evidence that LEU3 itself is under general amino acid control. Nucleic Acids Res. 13: 5261-5273. 


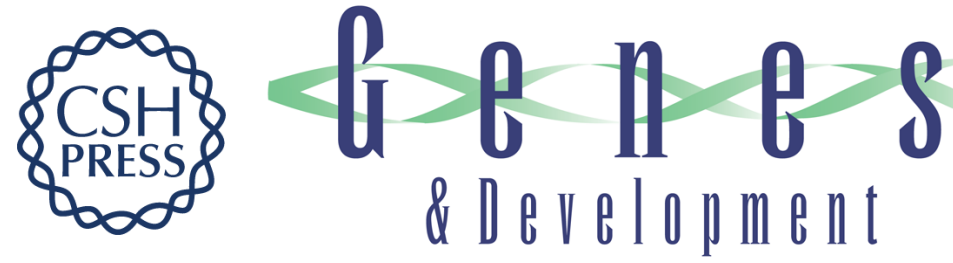

\section{Crystal structure of a PPR1-DNA complex: DNA recognition by proteins containing a Zn2Cys6 binuclear cluster.}

R Marmorstein and S C Harrison

Genes Dev. 1994, 8:

Access the most recent version at doi:10.1101/gad.8.20.2504

References This article cites 48 articles, 20 of which can be accessed free at:

http://genesdev.cshlp.org/content/8/20/2504.full.html\#ref-list-1

License

Email Alerting

Service

Receive free email alerts when new articles cite this article - sign up in the box at the top right corner of the article or click here.

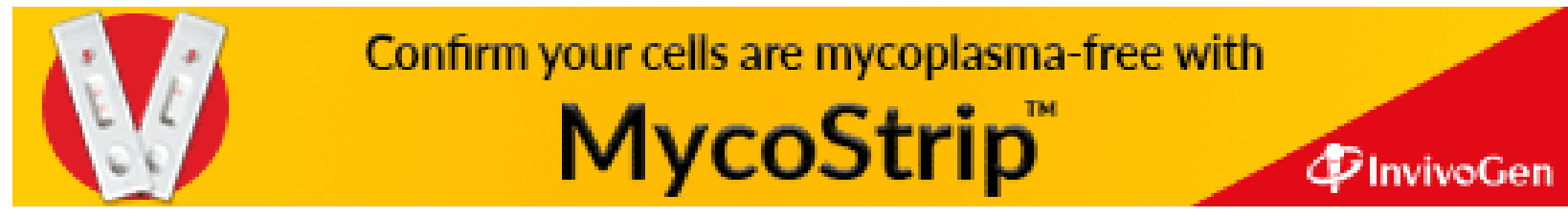

